laut Schmutzler in der Regel vor dem 40. Lebensjahr, abhängig vom Alter bei Diagnose des Erstkarzinoms. Bei BRCA2 lag das Alter bei Zweiterkrankung zwischen 40 und 50 Jahren. Zum Beispiel könne man bei einer 52-jährigen Brustkrebspatientin mit BRCA2-Mutation, einem lokalen Rezidivrisiko von $45 \%$ und einem kontralateralen Risiko von $11 \%$ mit der kontralateralen Mastektomie in der Regel noch abwarten.

Die Indikation zu einer prophylaktischen Mastektomie könne nur nach ausführlicher, nicht direktiver Beratung im Rahmen eines informierten Entscheidungsprozesses gestellt werden, erläuterte Schmutzler. Wichtig sei eine "verständliche Darstellung mit absoluten Zahlen zu einem überschaubaren Zeitraum". Dabei dürfe auch der Hinweis auf Nebenwirkungen bei einem großen Eingriff wie der Mastektomie nicht fehlen. Wie der plastische Chirurg Prof. Christoph Heitmann, München, betonte, gibt es bei der Operationsmethode keinen Goldstandard. Die onkologische Sicherheit steht im Vordergrund; zweite Priorität hat der Wunsch der Patientin. Es sei wichtig, alle vorhandenen Methoden anzubieten und auf der Grundlage des individuellen Phänotyps zu entscheiden. Bei der haut- bzw. mamillensparenden Mastektomie müsse man darauf achten, dass der Hautlappen gut durchblutet bleibt, um Nekrosen zu verhindern. Dass Dilemma besteht darin, dass man den Hautlappen extrem dünn wählen muss, um zu vermeiden, dass Reste von Brustgewebe verbleiben. Wichtig: Auch bei einer Nipplesparing-Mastektomie, wie sie Angelina Jolie erhalten hat, muss die Frau regelmäBig zur Kontrolle. Entscheidet man sich für ein Implantat, ist das Mittel der Wahl die Operation mit Matrix. Diese dient dazu, das Implantat, das am unteren Pol nicht vom M. pectoralis bedeckt ist, vollständig zu umhüllen. Bei der Sofortrekonstruktion mit Eigengewebe müsse man abwägen, welche Entnahmestelle im Einzelfall am besten geeignet ist. Oft kommt, vor allem bei nicht sehr schlanken Patientinnen, der Unterbauch infrage, alternativ das Gesäß.

Vor der Entscheidung für eine prophylaktische Mastektomie sei auch die psychische Belastungssituation zu berücksichtigen. „Es gibt fast nie akuten Zeitdruck", schloss Schmutzler. Man könne der Patientin also getrost genügend Zeit für eine sichere Entscheidung einräumen.

\section{Wenig Positives zum triple-negativen Karzinom}

Auf Kongressen, die sich mit Brustkrebs beschäftigen, gehört das Kapitel „triple-negatives Mammakarzinom“ meist zu den eher düsteren. Erfolgsmeldungen sind hier noch immer selten.

Eigentlich gibt es das triple-negative Mammakarzinom (TNMK) gar nicht. „Wir haben es mit einem Sammeltopf von Erkrankungen zu tun", sagte Dr. Cornelia Liedtke, Lübeck. Damit spann sie einen Faden fort, den eine Arbeitsgruppe um Lisa Carey von der University of North Carolina, Chapel Hill, vor rund drei Jahren ausgelegt hatte [Nat Rev Clin Oncol 2010; 7: 683-92]. Diese hatten sich ebenfalls die Frage gestellt, ob das triplenegative Mammakarzinom tatsächlich eine Krankheitsentität darstelle oder ob es sich nicht lediglich um eine bequeme Bezeichnung handle. Formal fallen auch Tumoren wie das adenoid-zystische Karzinom unter die Bezeichnung triple-negativ; doch das führt in die Irre, denn solche Tumoren haben eine ausgezeichnete Prognose. Bei den „echten“ triple-negativen Karzinomen ist es inzwischen nicht mehr leicht, zwischen all den Genexpressions- und Mutationsanalysen sowie der immunhistochemischen und molekularpathologischen Klassifizierungen die Orientierung zu bewahren. Prof. Carsten Denkert, Institut für Pathologie der Berliner Charité, formulierte es so: „Jeder einzelne Tumor ist anders" - und das heißt auch, jeder Tumor spricht anders an. Das erschwert neben der Therapie auch die Forschung, weil es schwieriger wird, genügend große Kohorten für die einzelnen Veränderungsmuster zu bilden.

Als triple-negativ gelten Tumoren, bei denen weniger als 1\% der Zellen Gene für Östrogen-, Progesteron- oder HER2/neu exprimieren. Davon betroffen sind rund 15\% der Brustkrebs-Patientinnen. Wichtigster prognostischer Faktor ist das Ansprechen auf die neoadjuvante Chemotherapie, betonte Liedtke. Denkert wies darauf hin, dass dies vor allem die lymphozytenreichen Tumoren betrifft. Insgesamt erreichen $22 \%$ der Patientinnen mit triple-negativem Karzinom eine pathologische Komplettremission. Bei den Rezeptor-positiven Krebsarten geschieht dies nur in 11\% der Fälle. Dennoch bleibt die Gesamtprognose beim triple-negativen Karzinom schlecht. Häufig treten schon in den ersten zweieinhalb bis drei Jahren prognostisch ungünstige Rezidive auf. Zielgerichtete Therapien, das betonten alle Vortragenden, sind nach wie vor nicht möglich. Außer der Chemotherapie verspricht allenfalls die Gabe des Angiogenesehemmers Bevacizumab Erfolg.

Ein weiterer Ansatz ist die Therapie mit Eribulin, einem nicht Taxan-basierten Zytostatikum. Wie Prof. Hans-Joachim Lück, Hannover, ausführte, könnte sich die Substanz aufgrund ihrer nur moderaten Nebenwirkungen besonders als Kombinationspartner eignen - etwa mit antiangiogenetisch wirksamen Präparaten. Hoffnungen setzen die Senologen auf den Einsatz von PARP-Inhibitoren, die sich gegen die Poly-ADP-RibosePolymerase richten.

Nicht übersehen werden sollte der Zusammenhang von triple-negativem Brustkrebs mit BRCA-Mutationen. Laut Angaben der Universitätsfrauenklinik Düsseldorf sind einerseits $80 \%$ der Mammakarzinome mit einer BRCA-1-Mutation triple-negativ. Andererseits finden sich bei $11 \%$ der Patientinnen unter 40 Jahren mit einem triple-negativen Karzinom Veränderungen von BRCA-1 oder BRCA-2 [Hüttemann U et al. Der Gynäkologe 2010; 43: 1002-7]. Liedtke berichtete, dass in den USA bereits allen Frauen, die vor dem 60. Lebensjahr an einem triple-negativen Mammakarzinom erkranken, eine genetische Testung und Beratung nahegelegt wird.

Dr. Robert Bublak

Weitere Meldungen vom Senologiekongress auf springermedizin.de

\footnotetext{
- Interdisziplinäre Zusammenarbeit war großgeschrieben auf der 33. Jahrestagung der DGS. Weitere Beiträge finden Sie in unserem Online-Dossier unter

www.springermedizin.de/senologiekongress-2013
} 